Pathologe $2020 \cdot 41: 515-522$

https://doi.org/10.1007/s00292-020-00790-z

Online publiziert: 26. Mai 2020

(C) Der/die Autor(en) 2020

Rubrikherausgeber

K. W. Schmid, Essen

H. A. Baba, Essen

Ewgenija Gutjahr' · Agathe Streng' · Sebastian Aulmann ${ }^{2}$.

Christa Flechtenmacher ${ }^{1} \cdot{\text { Ferdinand } \text { Toberer }^{3} \cdot \text { Jörg Heil }^{4} \cdot \text { Werner Böcker }}^{5}$.

Peter Sinn'

'Pathologisches Institut, Universitätsklinikum Heidelberg, Heidelberg, Deutschland

${ }^{2}$ Opti-Path, MVZ für Pathologie, Frankfurt, Deutschland

${ }^{3}$ Universitäts-Hautklinik, Heidelberg, Deutschland

${ }^{4}$ Universitäts-Frauenklinik, Heidelberg, Deutschland

${ }^{5}$ Dermatologische Gemeinschaftspraxis am Tibarg, Hamburg, Deutschland

\title{
Pathologie der Mamillenregion
}

\section{Teil Il: Tumoren, tumorartige Läsionen und Überschussbildungen}

\begin{abstract}
Tumoren und tumorartige Erkrankungen der Mamillenregion zeigen ein charakteristisches morphologisches Spektrum mit Besonderheiten im Vergleich zu den gewöhnlichen benignen und malignen Tumorerkrankungen der Mamma. Dieses betrifft die epithelialen Tumoren der Mamma, wozu das Mamillenadenom und der syringomatöse Tumor der Mamille gehören, als auch die nichtepithelialen Tumoren einschließlich dem malignen Melanom der Mamille und seltenen mesenchymale Tumoren der Mamille. In diesem Review diskutieren wir die relevanten Veränderungen, morphologischen Diagnosekriterien und die klinischen Konsequenzen, die sich aus der bioptischen Sicherung ergeben.
\end{abstract}

\section{Karzinome der Mamille}

Von der Mamille ausgehende oder rein intramamillär lokalisierte Karzinome sind selten und machen nur $1,2 \%$ aller Mammakarzinome aus [54], wobei es sich meist um gut differenzierte, stark hormonrezeptorpositive Karzinome ohne Bezug zu den großen Milchgängen handelt. Einzelne Fallberichte beschreiben ein intramamilläres solid-papilläres Mammakarzinom [60]. Intramamilläre Mammakarzinome können assoziiert sein mit einem Milchgangpapillom oder einem Mamillenadenom [66]. Ferner gilt die Mamillenregion als die bevorzug- te Lokalisation des Basalzellkarzinoms (Basalioms) der Mamma bei beiden Geschlechtern, wobei es sich meist um größere, lokal fortgeschrittene Tumoren handelt $[10,18]$. Klinisch präsentiert sich das Basalzellkarzinom der Mamille als eine erythematöse Läsion mit Schuppung, Ulzeration und subareolärem Tumor und gilt als aggressiver als in anderen Lokalisationen. Auch Plattenepithelkarzinome der Mamille sind beschrieben [16].

\section{Intramamilläres Papillom}

Die häufigste Läsion der zentralen Milchgänge ist das solitäre intraduktale $\mathrm{Pa}$ pillom, welches meist durch eine seröse oder, bei häufig herdförmig ausgebildeten Nekrosen des Papilloms, blutige Mamillensekretion klinisch manifest wird oder auch bei oberflächlicher Lage tastbar sein kann. Derhistologische Befund eines Milchgangpapilloms ist gekennzeichnet durch unterschiedlich breite, fibrosierte oder sklerosierte Papillenstöcke, die von einem zweireihigen Epithel mit basalem Myoepithel und luminalem, meist zylindrischem Drüsenepithel, nicht selten vom Typ einer Kolumnarzellmetasie, ausgekleidet werden. Die epithelialen Veränderungen umfassen die apokri-

„Teil I: „Pathologie der Mamillenregion; Morbus Paget der Mamille, Varianten und Differenzialdiagnosen" ist zu finden unter: https://doi.org/ 10.1007/s00292-020-00772-1. 

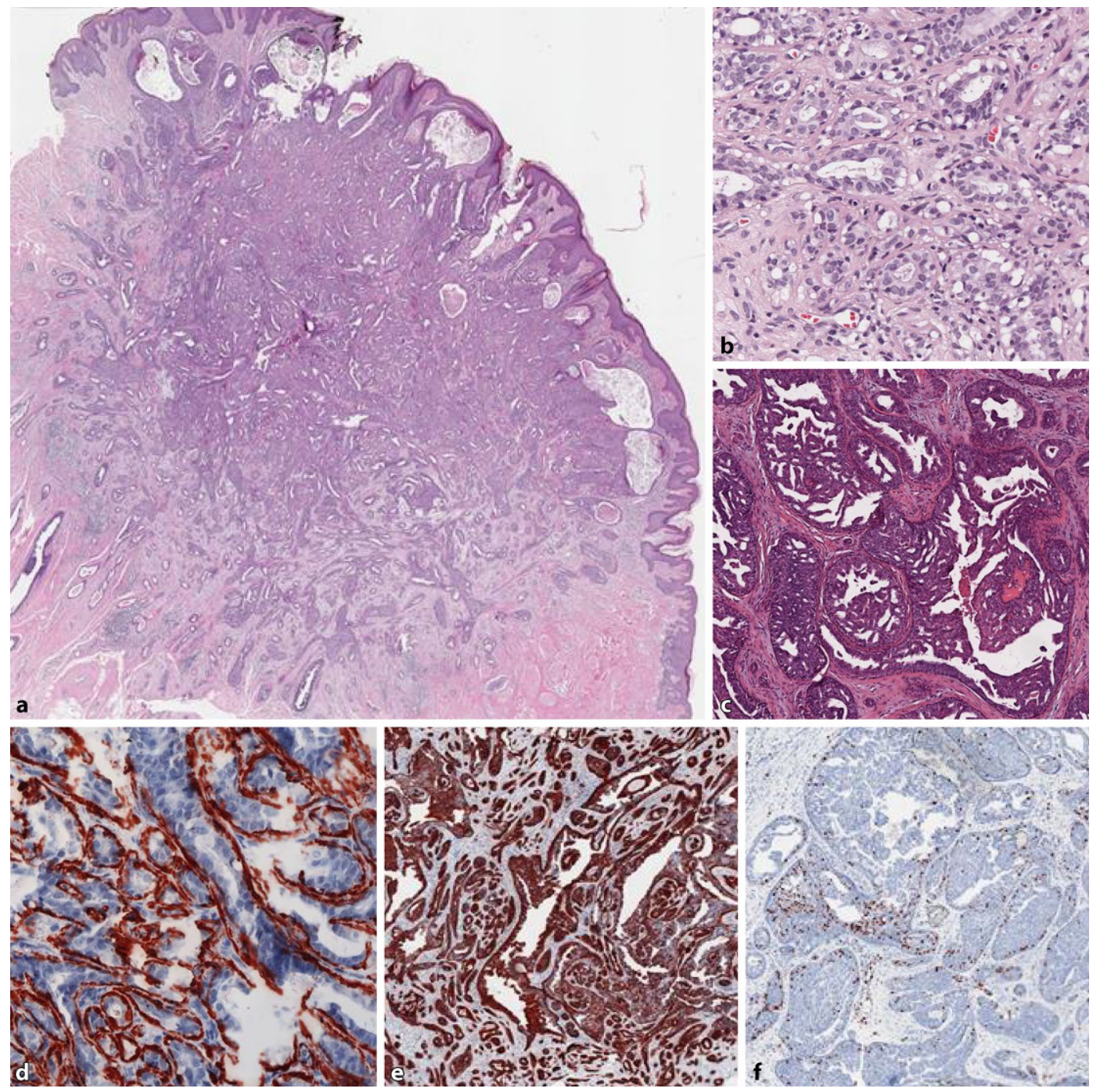

Abb. 1 Mamillenadenom. a Übersichtsvergrößerung mit komplexer adenomatöser Wuchsform und Bezug zur Epidermis. b, c Azinären bzw. papillomatöse Wachstumsmuster mit heterogenen Zellkernen. d, e Immunreaktivität für ASMA bzw. CK5/6 und fKi-67 mit niedriger Proliferationsaktivität

ne Metaplasie, unterschiedliche Formen der Adenosen sowie die einfache duktale Hyperplasie. Bei größeren Papillomen finden sich regressive Veränderungen mit Sklerose, seltener auch Plattenepithelmetaplasien oder Nekrosen. Beim intramamillären Papillom kann es zum partiellen Prolaps kommen.

\section{Mamillenadenom}

Das früher auch als erosive Adenomatose der Mamille, floride Papillomatose der Mamille oder subareoläre Papillomatose bezeichnete Mamillenadenom präsentiert sich klinisch in der Regel als kleiner, derber, oberflächlich gelegener knotiger Herdbefund ggf. mit persistierendem Erythem der Mamille oder der Areola oder, bei Erosion, als nässender Befund. Aufgrund der Lokalisation und der Symptomatik kann das Mamillenadenom klinisch für einen M. Paget der Mamille gehalten werden. Im Unterschied zum M. Paget fehlen jedoch meist ekzematöse Veränderungen. Betroffen sind Frauen aller Altersgruppen (20-87 Jahre, im Durchschnitt 43 Jahre), es wurden aber auch Einzelfälle bei Kindern und Jugendlichen $[11,59]$ oder bei Männern $[19,62]$ berichtet. Selten kommt ein bilaterales Auftreten von Mamillenadenomen vor. Bei Polythelie können Mamillenadenome in diesen überzähligen Brustwarzen [52] bzw. in einer akzessorischen Brustdrüse [53] auftreten.

Die Diagnose eines Mamillenadenoms ergibt sich aus der adenomatösen Struktur der Läsion mit dichtliegenden tubulären, duktulären, teilweise auch papillären Differenzierungsmustern. Das Mamillenadenom ist meist sehr ober- flächlich gelegen, mit Bezug zur Epidermis, eine Erosion ist nicht selten, welche klinisch zur Biopsie führt. Das Mamillenadenom ist häufig gut begrenzt, kann aber gelegentlich einen infiltrativen Rand aufweisen. Die auffälligen, soliden, fenestrierenden und teils mikropapillären Epithelproliferationen zeigen ein heterogenes Zell- und Kernbild mit kleinen, dicht liegenden Zellkernen und unscharf abgegrenzten Zytoplasma. Das Myoepithel ist verbreitert, zelluläre Atypien fehlen (• Abb. 1). Bei größeren Läsionen kann eine Sklerose des Stromas mit Architekturstörung hinzukommen, dennoch bleibt das organoides Grundmuster erhalten. Immunhistologisch können die epithelialen und myoepithelialen Zellen der Läsion unschwer mittels CK7 bzw. p63, CK5/6 oder CD10 differenziert werden [61]. 
Im Einzelfall kann die Abgrenzung eines Mamillenadenoms mit Epithelhyperplasie gegenüber einer atypischen Epithelhyperplasie oder einem duktalen Carcinoma in situ schwierig sein. Atypische Epithelhyperplasien mit Kernatypien, mikropapillärer Differenzierung, Mitosen und Komedonekrosen können in einem Mamillenadenom vorkommen, aber auch Übergänge in ein gut differenziertes duktales Carcinoma in situ [48]. Wegen der Häufigkeit florider Epithelhyperplasien in einem Mamillenadenom und der Ähnlichkeit zu atypischen epithelialen Proliferaten sollten diese aber immunhistochemisch abgesichert werden. Bei adenomatösem Wachstumsmuster und Sklerose kann ein invasives Mammakarzinom durch den Nachweis einer myoepithelialen Begrenzung der adenomatösen Drüsenkomplexe ausgeschlossen werden. Invasive Mammakarzinome, die mit einem Mamillenadenom assoziiert sind, sind selten $[20,27]$, ebenso Tumoren der Hautadnexe wie das noduläre Hidradenom [15, 57].

\section{Syringomatöser Tumor}

Die aktuelle WHO-Klassifikation grenzt die früher als Mamillenadenom vom syringomatösen Typ bezeichnete $\mathrm{Tu}$ morentität von den oben beschriebenen klassischen Mamillenadenomen ab und bezeichnet diesen als syringomatösen $\mathrm{Tu}$ mor der Nipple-/Areolaregion aufgrund der unscharfen Begrenzung, des infiltrativen Ausbreitungsmusters, einer Tendenz zur lokalen Rezidivierung und der fehlenden Ähnlichkeit zum Mamillenadenom (• Abb. 2). Trotz ihres infiltrativen Charakters verhalten sich diese Läsionen gutartig und eine vollständige lokale Exzision ist ausreichend.

Histologisch bildet der syringomatöse Mamillentumor infiltrierende kleindrüsige, tubuläre oder strangförmige Zellverbände. Die Tumordrüsen haben oft abgekantete, spitzwinklige Konturen, eine Tropfenform oder kommaförmige Fortsätze ähnlich wie die syringomatösen Tumoren der Haut. Die kleinen, isomorphen Zellkerne zeigen keine mitotische Aktivität. Eine perineurale Invasion kann beobachtet werden. Ein Bezug zu den

Pathologe 2020 -41:515-522 https://doi.org/10.1007/s00292-020-00790-z

(c) Der/die Autor(en) 2020

E. Gutjahr · A. Streng · S. Aulmann · C. Flechtenmacher · F. Toberer · J. Heil · W. Böcker · P. Sinn

Pathologie der Mamillenregion. Teil II: Tumoren, tumorartige Läsionen und Überschussbildungen

\section{Zusammenfassung}

Der Mamillen-Areola-Komplex ist Ausgangspunkt verschiedener morphologisch eigenständiger Tumoren und tumorartiger Veränderungen, die sich von den besonderen Strukturen der Mamille, insbesondere den intramamillären Milchgängen, den Anhangsgebilden und dem intramamillären Stroma ableiten lassen. Im Vordergrund stehen dabei epitheliale Tumoren gutartigen Verhaltens wie das Mamillenadenom und der syringomatöse Tumor der Mamille.
Seltener sind benigne mesenchymale Tumoren wie das Leiomyom der Mamille und tumorartige Veränderungen wie das Pseudolymphom der Mamillenregion. Bei den Überschussbildungen der Mamille sind die verschiedenen Formen der Polythelie und der Polymastie zu berücksichtigen.

Schlüsselwörter

Mamillenadenom · Syringomatöser Tumor . Pseudolymphom · Polythelie · Polymastie

\section{Pathology of the nipple-areola complex. Part II. Tumors, tumor- like lesions, and supernumerary breast lesions}

Abstract

The nipple-areola complex is the origin of various morphologically distinct tumors and tumor-like lesions, which can be delineated from the special structures of the nipple, in particular the intramammary ducts, skinappendages, and the intramammary stroma. Benign tumors are most frequent and this includes epithelial tumors such as mammary adenoma and syringomatous tumor of the nipple. Less commonly observed are benign mesenchymal tumors such as leiomyoma of the nipple, or tumor-like lesions like pseudolymphoma. With excess formations of the nipple, the different forms of polythelia and polymastia have to be considered.

\section{Keywords}

Nipple adenoma $\cdot$ Syringomatous tumor . Pseudolymphoma Polythelia Polymastia
Milchgängen oder der Epidermis besteht im Gegensatz zum Mamillenadenom in der Regel nicht. Das Vorhandensein einer Myoepithelschicht ist nicht ganz unumstritten, wird aber aufgrund der positiven Reaktion für die „Myoepithelzellmarker" p63 oder Ck5/6 in der Literatur mehrheitlich erwähnt [26, 39, 67]. Kürzlich ist jedoch aufgrund immunhistologischer Befunde gezeigt worden, dass es sich bei dem syringomatösen Tumor der Mamille - analog zum Syringom der Haut - im Kern um eine Proliferation p63+Ck5/14+ Progenitorzellen handelt, die sich zu glandulären (Ck8/18) und squamösen Zellen (Ck10/13) differenzieren. Weitaus seltener und zumeist nur fokal findet man im syringomatösen Tumore der Mamille Differenzierungen zu myoepithelialen Zellen (Aktin, CD10, Calponin etc.), die im Syringom der Haut nicht zu beobachten sind [6]. Im Kontext mit dem syringomatösen Tumor kann die Expression von p63 und hochmolekularen Zytokeratinen Ck5 und/oder Ck14 somit nicht als Hinweis für die myoepitheliale Differenzierung in diesem Tumor herangezogen werden. Allerdings hilft die Expression dieser Marker und die fehlende Expression von Hormonrezeptoren sowie auch das Fehlen einer intraduktalen Tumorkomponente in der Abgrenzung des syringomatösen Mamillentumors vom tubulären Mammakarzinom (p63 negativ, Ck5/14 negativ, Aktinve, $\mathrm{ER}+$ ).

Syringomatöse Tumoren ähneln in verschiedenen Aspekten dem niedrigmalignen adenosquamösen Karzinom der Mamma, das zu den metaplastischen Mammakarzinomen vom Speicheldrüsentyp gerechnet wird und zumindest teilweise ebenfalls eine myoepitheliale Zellschicht aufweist [29, 42, 44]. 

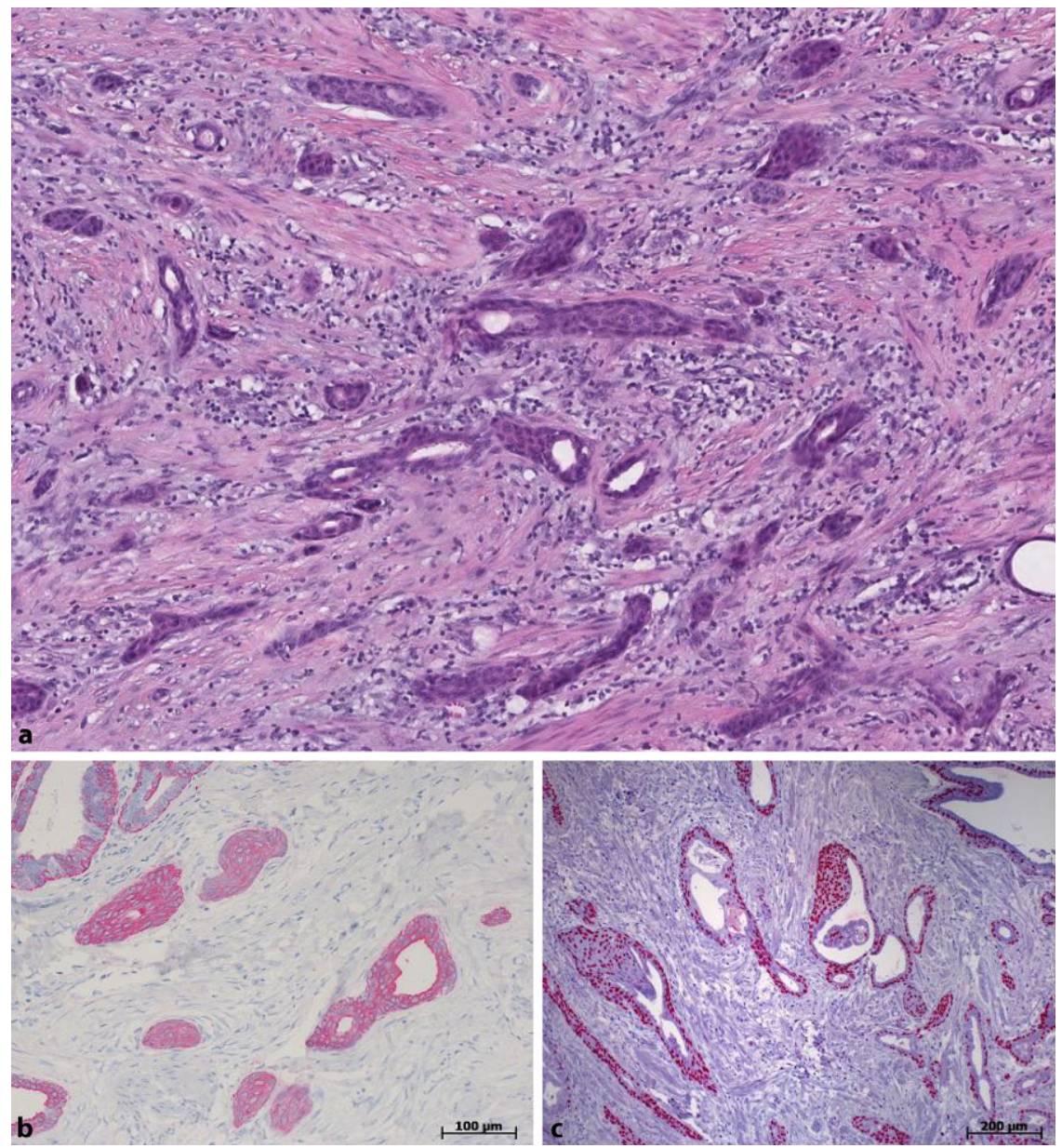

Abb. 2 \ Syringomatöser Tumor. a Typischer Aspekt mit adenomatösem Wuchsmuster und plattenepithelialer Differenzierung. b Starke Immunreaktion für CK5. c Partielle Positivität für p63

Aufgrund der ähnlichen Eigenschaften erscheint unklar, ob das niedrigmaligne adenosquamösen Karzinom und der syringomatöse Mamillentumor eine oder zwei verschiedene Entitäten darstellen [7]. Das Hauptunterscheidungsmerkmal beider Tumoren ist die Lage in der Brust: Syringomatöse Tumoren entstehen in der Mamille und infiltrieren sekundär das subareoläre Mammaparenchym, während das niedrigmaligne adenosquamösen Karzinom peripherer im Brustparenchym auftritt [7].

\section{Mesenchymale Tumoren}

$\mathrm{Zu}$ den mesenchymalen Tumoren der Mamille und der Areola, die gelegentlich beobachtet werden, gehört insbesondere das Leiomyom der Mamille. Der M. areolaris mamillaris, der als Ausgangspunkt gilt, gehört, ebenso wie die Mm. arrectores pilae, zu den Hautmus-

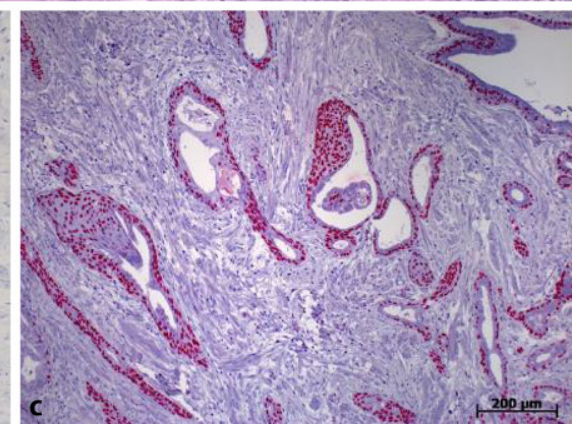

\section{Malignes Melanom}

Maligne Melanome der Mamille sind eine Rarität, und auch auf der Brusthaut können primäre maligne Melanome vereinzelt auftreten [3, 32]. Dagegen gehören Metastasen eines malignen Melanoms zu den häufigsten Metastasen von extramammären Malignomen in die Brust und machen etwa $22 \%$ der Fälle aus [13]. Fallberichte beschreiben auch primäre, nichtkutane Melanome der Mamma [43, 64].

Zum malignen Melanom der Mamille sind verschiedene Kasuistiken publiziert und wir haben ebenfalls einen solchen Fall beobachtet, welcher mit einer lentiginösen Tumorkomponente assoziiert war (•Abb. 3). Es wird sowohl von prämenopausalen als auch von postmenopausalen Patientinnen berichtet $[31,36]$. Differenzialdiagnostisch muss vor allem ein M. Paget ausgeschlossen werden, zumal beim M. Paget der Mamille selten auch pigmentierte Varianten vorliegen können [2, 45]. Differenzialdiagnostisch kann aber auch bei Mammakarzinomen gelegentlich eine Pigmentierung beobachtet werden, die auf einer Phagozytose von Melanin oder Lipofuszin beruht [49, 51].

\section{Pseudolymphom der Mamille}

keln des Mamillen-Areola-Komplexes und umfasst radiäre (Meyerholz) und zirkuläre (Sappey) glatte Muskelfasern des Warzenhofs. Das Leiomyom kommt sowohl intramamillär als auch im Warzenhof vor, da die Fasern des M. areolaris in die Mamille hineinziehen und hier eine dünne subkutane Schicht bilden [68]. Ebenso wie die ortsständige glatte Muskulatur ist das Leiomyom meist positiv für die Östrogen- und Progesteronrezeptoren [9]. Leiomyosarkome der Mamille sind demgegenüber selten [24]. Bei der Typ-I-Neurofibromatose mit Befall des Mamillen-Areola-Komplexes finden sich extensive plexiforme Neurofibromen, die plastisch-chirurgische Eingriffe erfordern $[8,21,33]$. Seltene mesenchymale Tumoren der Mamille betreffen das Angiomyxom [65] und das intramamilläre Hämangiom.
Das Pseudolymphom der Mamma ist ein gutartiger lymphoproliferativer Prozess, der einem Marginalzonenlymphom ähnelt, ohne die hierfür charakteristischen nisch zeigen typische Fälle eine Rötung und Schwellung der Mamillenregion, bei weiter fortgeschrittenen Läsionen können auch oberflächliche Erosionen der Epidermis auftreten. Der Begriff des Pseudolymphoms geht auf Ackermann zurück (Zit. n. [56]), ältere Synonyme für das kutane Pseudolymphom der Mamille sind: Lymphocytoma cutis, Lymphadenosis benigna cutis (Bäfverstedt) [30] oder follikuläre lymphatische Hyperplasie [50]. Die Entstehung dieser kutanen Pseudolymphome kann durch eine Reihe antigener Stimuli ausgelöst werden. Neben der besonders häufigen Assoziation mit Borrelia burgdorferi [12, 35] wurden ähnliche Veränderungen auch nach Kriterien voll ausgebildet zu haben. Kli- 

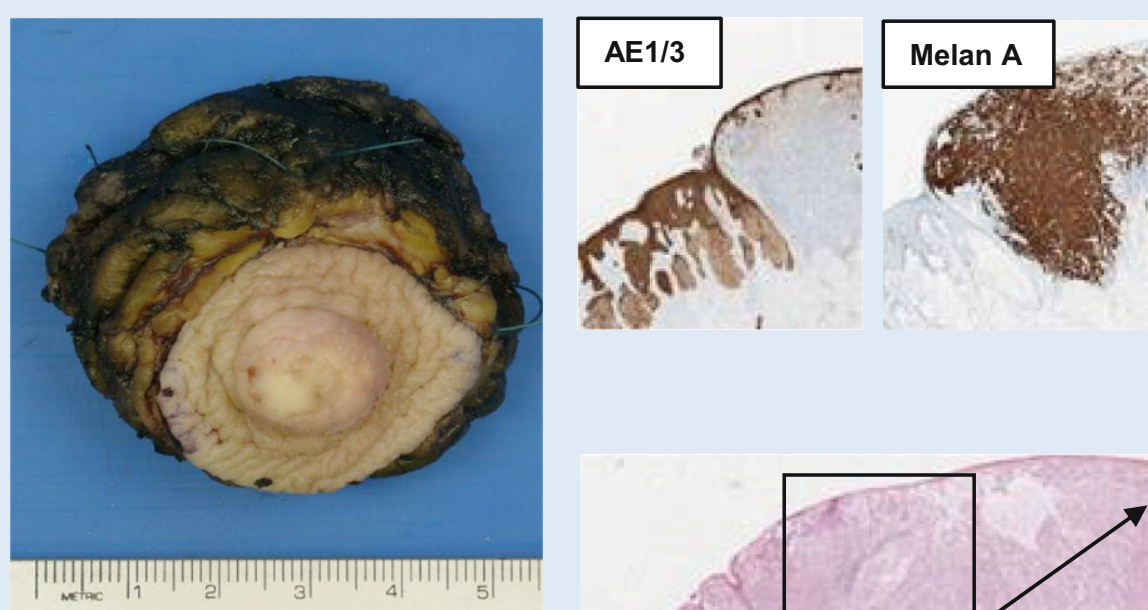

a

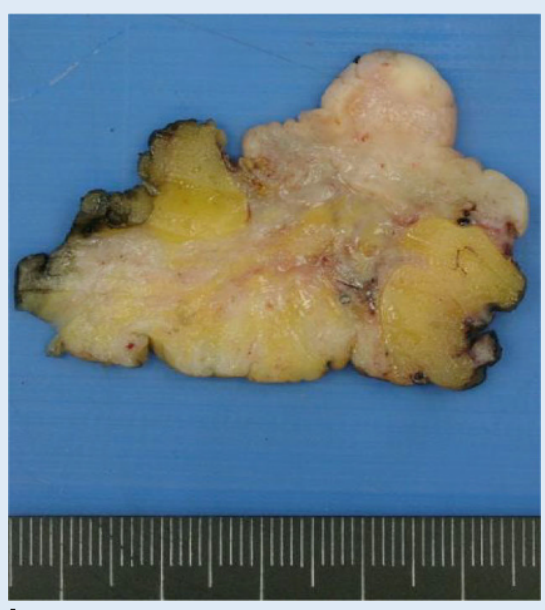

b

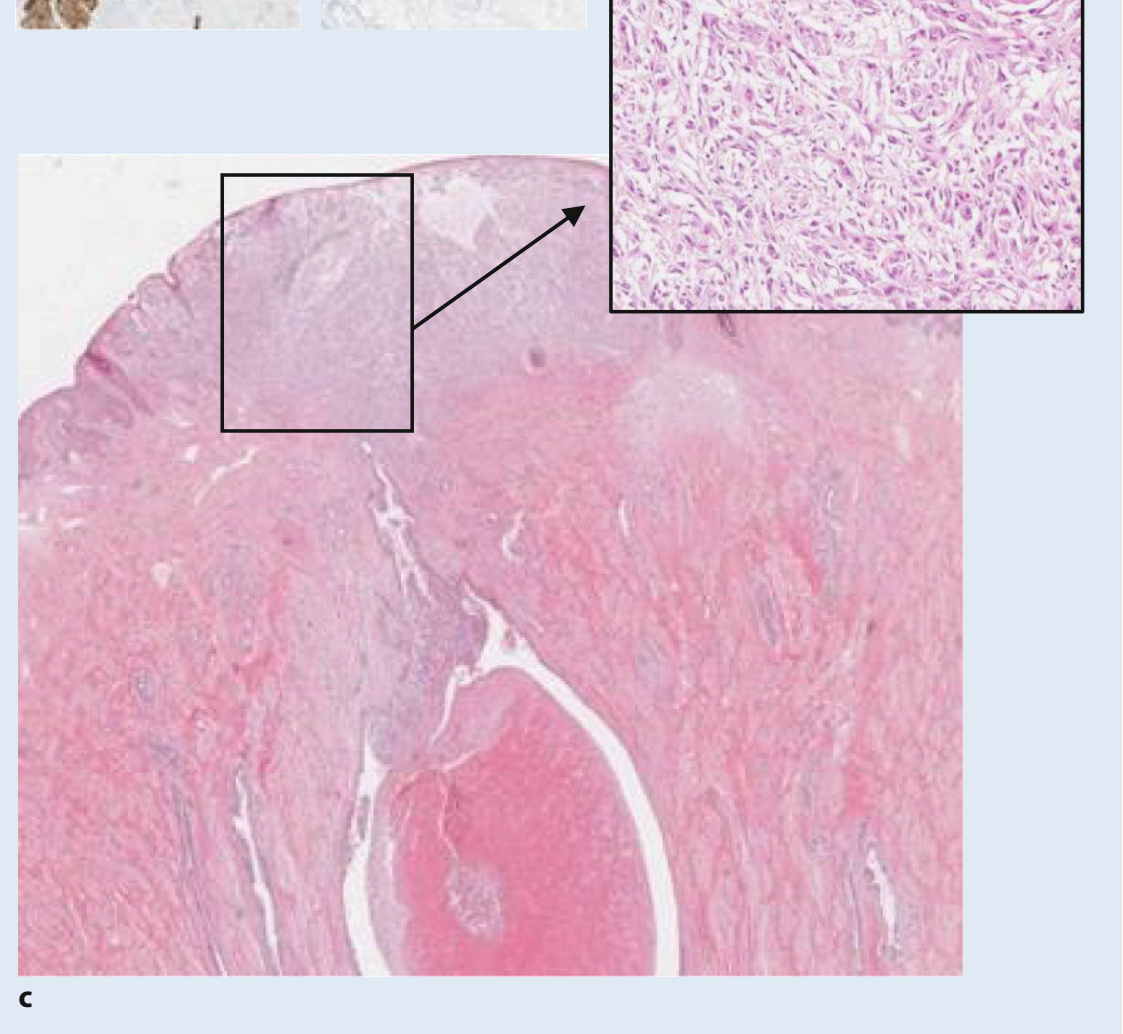

Abb. 3 A Malignes Melanom der Mamille.a,b Im Bereich derMamillezeigtsich makroskopischeine weißliche, knotige Läsion. c Eine von der Epidermis der Mamille ausgehende unpigmentierte Neoplasie mit deutlicher Pleomorphie. c Negativer Befund für Zytokeratine und homogen kräftige Immunreaktion gegen Melan A

anderen Insektenstichen, als Folge einer Behandlung mit Blutegeln, als Medikamentenreaktion, nach Impfungen, aber auch Akupunkturbehandlungen sowie Tätowierungen beschrieben $[46,55,57$, 58]. Die Mamille ist neben den Ohrmuscheln und dem Skrotum einer der charakteristischen Prädilektionsstellen für borrelienassoziierte Pseudolymphome [12].

Histologisch ist ein gemischt knotiges und diffuses Infiltrat, oft mit keilförmiger Ausbreitung innerhalb der Dermis sowie der oberflächlichen Subkutis typisch. Die in der Läsion enthaltenen Keimzentren haben eine Tendenz zur Konfluenz, Mantelzonen können fehlen. Daneben sind zumeist auch Plasmazellen, eosinophile
Granulozyten und distinkte T-Zell-Populationen nachweisbar, typisch ist eine Grenzzone mit ausgesparter Epidermis. Immunfärbungen für CD21 oder CD23 markieren ein regelhaftes, organoides Netzwerk follikulärer dendritischer Zellen. Molekulare Klonalitätstestungen ergaben in der Literatur zumeist (aber nicht in allen Fällen) polyklonale IgHAmplifikate [35, 47].

Klinisch können sich Pseudolymphome im Verlauf von Monaten und Jahren spontan zurückbilden, kleinere Knoten werden häufig auch lokal exzidiert. Bei nachgewiesener Borrelieninfektion kann in einem Teil der Fälle durch eine Antibiotikatherapie auch eine Remission der assoziierten Pseudolymphome induziert werden. Weitere Behandlungsmöglichkeiten, die in einem Teil der Fälle zu einer Befundremission führen sind lokale Injektionen von Corticosteroiden oder Interferon-alpha. In seltenen Fällen können Borrelien auch systemische Pseudolymphome auslösen, die sich nach Antibiotikatherapie zurückbilden [1].

\section{Polythelie und Polymastie}

Akzessorisches mammäres Gewebe, entweder in Form einer Polymastie oder einer Polythelie findet sich bei Frauen in $1-5 \%$ und in einem ähnlichen Prozentsatz auch bei Männern [14, 23]. Man spricht von einer Polymastie bei Vorhandensein von mammärem Drüsengewebe 


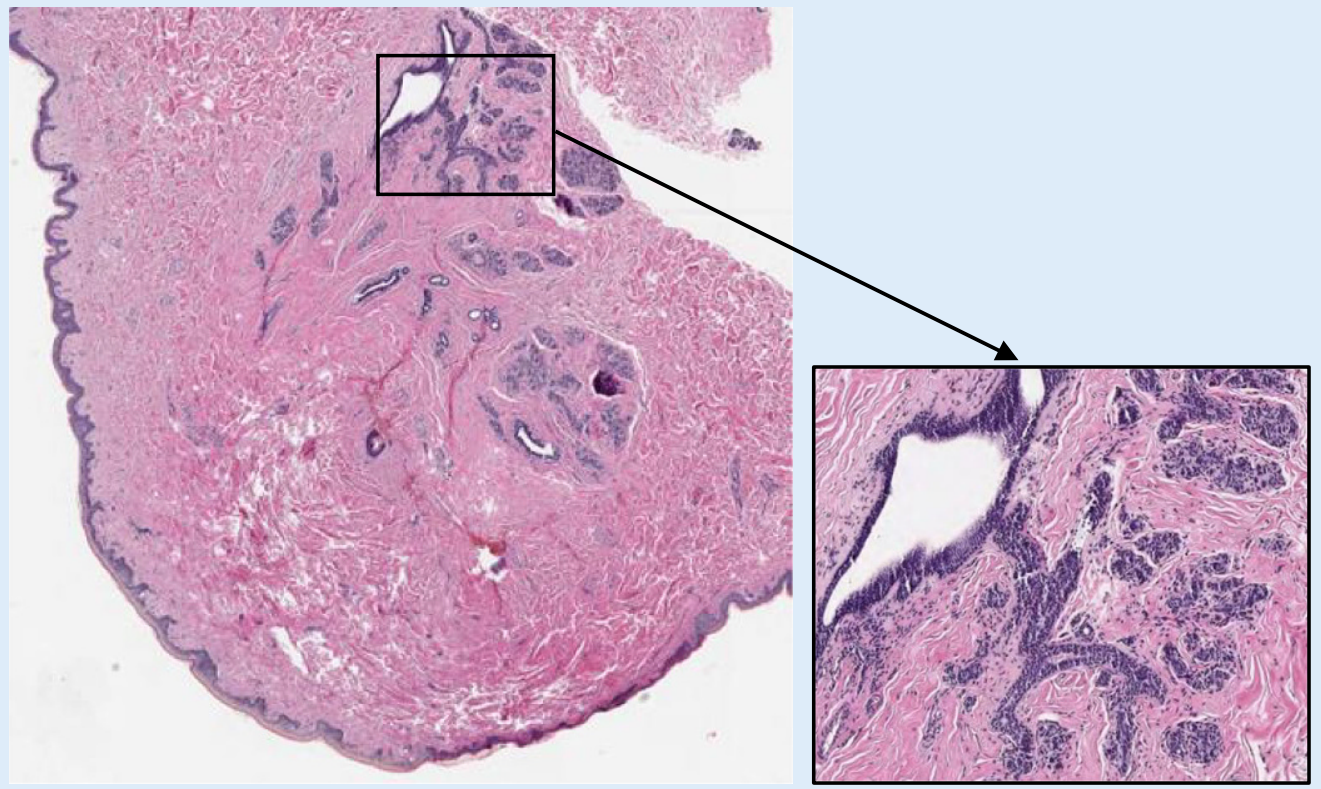

a

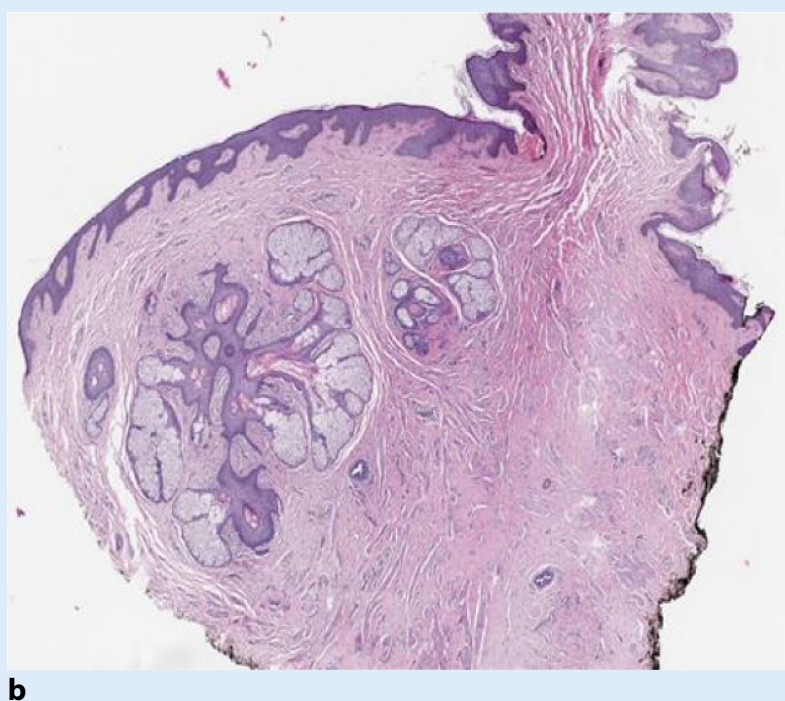

Abb. $4<$ a Die Polythelia completa mit regelrecht ausgebildeter Anlage einer Mamille samt Areola und im vorliegenden Fall auch einzelne Azini (im Inlet dargestellt), jedoch nicht das Vollbild einer Mamma accessoria. b Im Gegensatz zur Polythelia completa zeigen sich bei der Polythelia pilosa lediglich einzelne Haarfollikelaggregate in typischer Lokalisation (mit oder ohne Mamille) oder von einer Polythelie bei akzessorischer Mamille und/oder einer Areola ohne Drüsengewebe (•Abb. 4). Akzessorisches Drüsengewebe kommt meist oberhalb der regulären Mammae und am häufigsten in der Axilla vor, wobei man auch von Mamma aberrans bzw. Mamma accessoria spricht. Bei ektoper Lokalisation außerhalb der Milchleiste (z. B. inguinal oder am Oberschenkel) wird der Begriff „Pseudomamma“ verwendet [38]. Eine familiäre Häufung des Auftretens ektopen mammären Gewebes ist beschrieben [63], ebenso damit assoziierte Tumoren [17] sowie eine Korrelation mit chroni- schen Nierenerkrankungen [37]. Die Polythelie tritt ober- oder unterhalb einer Mamma auf, auch bilateral, in der Regel im Bereich der hypothetischen embryonalen Milchleiste, die von der Axilla zur Inguinalregion zieht [5] und gilt daher als „Atavismus“, d. h. als Wiederauftreten eines bei Tieren gefundenen Zustandes [34]. Es sind 3 Fälle von Frauen mit kompletter Milchleiste und jeweils $10 \mathrm{Ma}$ millen beschrieben [22, 40, 41]. Weitere, weniger gut belegte Fälle finden sich in der älteren Literatur. Die Polythelie kann klinisch und histologisch in 4 Formen eingeteilt werden $[4,28]$, wobei die Polythelia completa die häufigste Form der Polythelie darstellt. Zur Klassifikation und Häufigkeiten siehe • Tab. 1.

\section{Fazit für die Praxis}

- Primäre Karzinome der Mamille betreffen als seltene Primärlokalisation das Mammakarzinom, aber auch Hauttumoren wie das Basaliom der Mamille.

- Zu den benignen Tumoren der Mamille gehören das Milchgangpapillom, das Mamillenadenom und der syringomatöse Mamillentumor.

- Der häufigste mesenchymale Tumor der Mamille ist das Leiomyom, 
Tab. 1 Einteilung und Häufigkeiten der Polythelien und Polymastien. (Nach Bässler [4], Horn [25] und Kajava [28]

\section{Merkmale}

\section{Polythelie}

Polythelia completa

Häufigste Form der Polythelie mit Mamille, Ausführungsgängen und Areola, aber ohne Drüsenparenchym. In der Areola können Talgdrüsen und Haare auftreten, ferner glatte Faserbündel des M. areolaris

Polythelia mamillaris Zweithäufigste Form mit Merkmalen wie bei der Polythelia completa, jedoch ohne Anlage einer Areola

Polythelia areolaris

Polymastia

Polymastia completa

(Mamma accessoria)

Polymastia mamillaris

Polymastia areolaris

Polymastia glandularis (Mamma aberrans)
Anlage der Brustwarze ohne Mamille oder Gangstrukturen. Die areoläre Epidermis weist eine Pigmentierung, Haare, Talgdrüsen und Schweißdrüsen auf. Das Vorkommen von Bündeln des $M$. areolaris gilt als sicheres Merkmal der Polythelia areolaris

Eine Haargruppe, die an typischer Stelle sitzt, öfter bei erwachsenen Männern anzutreffen

Völlig ausgebildete, aber kleinere akzessorische Milchdrüse

Nur Mamille vorhanden, ohne Warzenhof, mit Drüsensub-

Warzenhof ohne Mamille

Keine Mamille und kein Warzenhof, nur Drüsen. Vorkommen meist in der Axilla stanz in der Tiefe
Selten

Selten

$80,4 \%$

Häufig

keit

$63,9 \%$

$16,9 \%$

$10,9 \%$

$80,4 \%$

\section{welches sich intramamillär oder areolär manifestiert und von den $\mathrm{Mm}$. areolaris ausgeht. \\ - Das primäre maligne Melanom betrifft sporadisch auch die Mamille und kann eine nichtinvasive lentiginöse oder pagetoide Übergangszone aufweisen. \\ - Das Pseudolymphom der Mamillenre- gion beruht meist auf einer Borreliose und führt zu einer unilateralen Rö- tung und Schwellung der Mamille. \\ - Die Überschussbildungen mit rudi- mentärer Anlage der Mamille und des Drüsenkörpers (Polythelie, Poly- mastie, Mamma aberrans) kommen in der Milchleiste vor, seltener auch an anderen Lokalisationen.}

\section{Korrespondenzadresse}

Prof. Dr. Peter Sinn

Pathologisches Institut, Universitätsklinikum Heidelberg

Im Neuenheimer Feld 224, 69120 Heidelberg,

Deutschland

peter.sinn@med.uni-heidelberg.de

Funding. Open Access funding provided by Projekt DEAL.

\section{Literatur}

1. Aigelsreiter A, Pump A, BuchhäusI W et al (2005) Successful antibiotic treatment of Borreliosis associated pseudolymphomatous systemic infiltrates. JInfect 51:e203-e206

2. Al-Daraji WI, O'Shea AM, Looi LM et al (2009) Pigmented mammary Paget's disease: not a melanoma. Histopathology 54:614-617

3. Alzaraa A, Sharma N (2008) Primary cutaneous melanoma of the breast: a case report. Cases J 1:212

4. Bässler R (1978) Pathologie der Brustdrüse. Springer, Berlin, New York

5. Boas H (1955) Über das Vorkommen der Mammae accessoriae. Untersuchung von 6456 Patienten. Hautarzt 6:253-256

6. Boecker W, Stenman G, Loening T et al (2015) Squamous/epidermoid differentiation in normal breast and salivary gland tissues and their corresponding tumors originate from $\mathrm{p} 63 / \mathrm{K} 5 / 14$ positive progenitor cells. Virchows Arch 466:21-36

7. Boecker W, Stenman G, Loening T et al (2014) Differentiation and histogenesis of syringomatous tumour of the nipple and low-grade adenosquamous carcinoma: evidence for a common origin. Histopathology 65:9-23

8. Bongiorno MR, Doukaki S, Arico M (2010) Neurofibromatosis of the nipple-areolar area: a case series. J Med Case Rep 4:22

9. Chaudhary KS, Shousha S (2004) Leiomyoma of the nipple, and normal subareolar muscle fibres, are oestrogen and progesterone receptor positive. Histopathology 44:626-628

10. Chun KA, Cohen PR (2016) Basal cell carcinoma of the nipple-areola complex: a comprehensive review of the world literature. Dermatol Ther (Heidelb) 6:379-395

11. Clune JE, Kozakewich HP, Vanbeek CA et al (2009) Nipple adenoma in infancy. J Pediatr Surg 44:2219-2222

12. Colli C, Leinweber B, Müllegger R et al (2004) Borrelia burgdorferi-associated lymphocytoma cutis: clinicopathologic, immunophenotypic, and molecular study of 106 cases. J Cutan Pathol 31:232-240

13. Delair DF, Corben AD, Catalano JP et al (2013) Nonmammary metastases to the breast and axilla: a study of 85 cases. Mod Pathol 26:343-349

14. Dixon JM, Mansel RE (1994) ABC of breast diseases. Congenital problems and aberrations of normal breast development and involution. BMJ 309:797-800

15. Domoto H, Terahata S, Sato Ket al (1998) Nodular hidradenoma of the breast: report of two cases with literature review. Pathol Int 48:907-911

16. Dye K, Saucedo M, Raju D et al (2017) A common cancer in an uncommon location: a case report of squamous cell carcinoma of the nipple. Int J Surg Case Rep 36:94-97

17. Fama F, Cicciu M, Sindoni A et al (2016) Prevalence of ectopic breast tissue and tumor: a 20-year single center experience. Clin Breast Cancer 16:e107-e112

18. Ferguson MS, Nouraei SA, Davies BJ et al (2009) Basal cell carcinoma of the nipple-areola complex. Dermatol Surg 35:1771-1775

19. Fernandez-Flores A, Suarez-Peñaranda JM (2011) Immunophenotype of nipple adenoma in a male patient. Appl Immunohistochem Mol Morphol 19:190-194

20. Gobbi H, Simpson JF, Jensen RA et al (2003) Metaplastic spindle cell breast tumors arising 
within papillomas, complex sclerosing lesions, and nipple adenomas. Mod Pathol 16:893-901

21. Goksugur N, Gurel S (2011) Neurofibromatosis of nipple-areola complex. Breast J 17:424

22. Graham-Campbell R (1936) Polythelia. Br Med J 1:471-472

23. GrossI NA (2000) Supernumerary breast tissue: historical perspectives and clinical features. South Med J93:29-32

24. Guedes De La Puente X (2018) Leiomyosarcoma of the nipple-areola complex. In: Shiffman MA (Hrsg) Nipple-areolar complex reconstruction. Principles and clinical techniques. Springer, Berlin, Heideberg, New York, S 181-184

25. Horn J (1924) Hypermastia axillaris: klinische und anatomische Untersuchungen. Acta Obstet Gynecol Scand 3:1-126

26. Ichinokawa Y, Ohtuki A, Hattori Metal (2012) A case of syringomatous adenoma of the nipple. Case Rep Dermatol 4:98-103

27. Jones M, Tavassoli $F$ (1995) Coexistence of nipple duct adenoma and breast carcinoma: a clinicopathologic study of five cases and review of the literature. Mod Pathol 8:633-636

28. Kajava Y (1915) Ylilukuisten nisien esiintymisestä suomalaisilla [Das Vorkommen von überzähligen Brustwarzen bei den Finnen]. Duodecim XXXI:143-170

29. Kawaguchi K, Shin SJ (2012) Immunohistochemical staining characteristics of low-grade adenosquamous carcinoma of the breast. Am J Surg Pathol 36:1009-1020

30. Kindermann G, Seibicke E (1977) Lymphadenosis benigna cutis (Bafverstedt) im Warzenbereich. Geburtshilfe Frauenheilkd 37:1024-1027

31. Kinoshita S, Yoshimoto K, Kyoda S et al (2007) Malignant melanoma originating on the female nipple: a case report. Breast Cancer 14:105-108

32. Kurul S, Tas F, Buyukbabani N et al (2005) Different manifestations of malignant melanoma in the breast: a report of 12 cases and a review of the literature.Jpn J Clin Oncol 35:202-206

33. Lannon DA, Harris PA, Butler PE (2003) Treatment of severe neurofibromatosis of the nipple-areola complex. Plast Reconstr Surg 111:952-953

34. Leichtenstern O (1878) Ueber das Vorkommen und die Bedeutung supernumerärer (accessorischer) Brüste und Brustwarzen. Virchows Arch 73:222-256

35. Leinweber B, Colli C, Chott A etal (2004) Differential diagnosis of cutaneous infiltrates of $B$ lymphocytes with folliculargrowth pattern. Am JDermatopathol 26:4-13

36. Lin CH, Lee HS, Yu JC (2007) Melanoma of the nipple mimicking Paget's disease. Dermatol Online J 13:18

37. Loukas M, Clarke P, Tubbs RS (2007) Accessory breasts: a historical and current perspective. Am Surg 73:525-528

38. Marinopoulos S, Arampatzisl,ZagouriFetal (2015) Pseudomamma of the inguinal region in a female patient: a case report. Int J Surg Case Rep 12:71-74

39. Montgomery ND, Bianchi GD, Klauber-Demore N et al (2014) Bilateral syringomatous adenomas of the nipple: case report with immunohistochemical characterization of a rare tumor mimicking malignancy. Am J Clin Pathol 141:727-731

40. Neugebauer FL (1886) Eine bisher einzig darstehende Beobachtung von Polymastie mit 10 Brustwarzen. Zentralbl Gynäkol 10:729-736

41. Pinkus $F$ (1927) Die normale Anatomie der Haut. In: Block B, Pinkus F, Spalteholz W (Hrsg) Anatomie der Haut. Handbuch der Haut- und
Geschlechtskrankheiten, Bd. 1. Springer, Berlin, S 1-378

42. Rakha EA, Coimbra ND, Hodi Z et al (2017) Immunoprofile of metaplastic carcinomas of the breast. Histopathology 70:975-985

43. Rassouli M, Voutsadakis IA (2016) Primary noncutaneous malignant melanoma of the breast. Breast J 22:688-691

44. Reis-Filho JS, Milanezi F, Paredes J et al (2003) Novel and classic myoepithelial/stem cell markers in metaplastic carcinomas of the breast. Appl Immunohistochem Mol Morphol 11:1-8

45. Requena L, Sangueza M, Sangueza OP et al (2002) Pigmented mammary Paget disease and pigmented epidermotropic metastases from breast carcinoma. Am J Dermatopathol 24:189-198

46. Rijlaarsdam JU, Bruynzeel DP, Vos W et al (1988) Immunohistochemical studies of lymphadenosis benigna cutis occurring in a tattoo. Am J Dermatopathol 10:518-523

47. Rijlaarsdam U, Bakels V, Van Oostveen JW et al (1992) Demonstration of clonal immunoglobulin gene rearrangements in cutaneous B-cell lymphomas and pseudo-B-cell lymphomas: differential diagnostic and pathogenetic aspects. J Invest Dermatol 99:749-754

48. Rosen PP, Hoda SA, Brogi E et al (2014) Rosen's breast pathology, 4. Aufl. Wolters Kluwer Health/ Lippincott Williams \& Wilkins, Philadelphia

49. Saitoh K, Saga K, Okazaki M et al (1998) Pigmented primary carcinoma of the breast: a clinical mimic of malignant melanoma. Br JDermatol 139:287-290

50. Schmid U, Eckert F (1989) Das Pseudolymphom-reaktive lymphatische Hyperplasie oder Prälymphom? Eine Übersicht mit besonderer Berücksichtigung des MALT. Pathologe 10:332-339

51. Shin SJ, Kanomata N, Rosen PP (2000) Mammary carcinoma with prominent cytoplasmic lipofuscin granules mimicking melanocytic differentiation. Histopathology 37:456-459

52. Shinn L, Woodward C, Boddu S et al (2011) Nipple adenoma arising in a supernumerary mammary gland: a case report. Tumori 97:812-814

53. Shioi Y, Nakamura S, Kawamura S et al (2012) Nipple adenoma arising from axillary accessory breast: a case report. Diagn Pathol 7:162

54. Siotos C, Mccoll M, Psoter K et al (2018) Tumor site and breast cancer prognosis. Clin Breast Cancer 18:e1045-e1052

55. Smolle J, Cerroni L, Kerl H (2000) Multiple pseudolymphomas caused by Hirudo medicinalis therapy. J Am Acad Dermatol 43:867-869

56. Smyczek-Gargya B, Geppert M (1994) Pseudolymphoma of the nipple. Eur J Obstet Gynecol Reprod Biol 53:139-141

57. Spyropoulou GA, Pavlidis L, Trakatelli Met al (2015) Rare benign tumours of the nipple. J Eur Acad Dermatol Venereol 29:7-13

58. Stavrianeas NG, Katoulis AC, Kanelleas A etal (2002) Papulonodular lichenoid and pseudolymphomatous reaction at the injection site of hepatitis $B$ virus vaccination. Dermatology 205:166-168

59. Sugai M, Murata K, Kimura N et al (2002) Adenoma of the nipple in an adolescent. Breast Cancer 9:254-256

60. Tacchini D, Vassallo L, Butorano MA et al (2016) Solid papillary carcinoma of the nipple: an in situ carcinoma or an expansive growth tumor? Pathologica 108:136-139

61. Tse GM, Tan PH, Lui PC et al (2007) The role of immunohistochemistry for smooth-muscle actin p63, CD10 and cytokeratin 14 in the differential diagnosis of papillary lesions of the breast. J Clin Pathol 60:315-320

62. Tuveri M, Calo PG, Mocci C et al (2010) Florid papillomatosis of the male nipple. Am J Surg 200:e39-e40

63. Urbani CE, Betti R (1995) Familial aberrant mammary tissue: a clinicoepidemiological survey of 18 cases. Dermatology 190:207-209

64. Vergine M, Guy C, Taylor MR (2015) Phyllodes tumor of the breast with malignant melanoma component: a case report. Int J Surg Pathol 23:483-488

65. Victoria Martínez AM, Sánchez Carazo JL, Alegre De Miquel V (2016) Superficial angiomyxoma of the nipple: a case report of an infrequent cutaneous tumour. Dermatol Online J 22(10):8

66. Wilsher MJ, Desai AJ, Pinder SE (2019) Low-grade adenosquamous carcinoma arising in association with a nipple adenoma. Histopathology. https:// doi.org/10.1111/his.14033

67. Yosepovich A, Perelman M, Ayalon S et al (2005) Syringomatous adenoma of the nipple: a case report. Pathol Res Pract 201:405-407

68. Zucca-Matthes G, Urban C, Vallejo A (2016) Anatomy of the nipple and breast ducts. Gland Surg 5:32-36 